\title{
Therapeutic Hemapheresis
}

\author{
Behrouz Mansouri Taleghani $^{\mathrm{a}}$ Erwin Strasser ${ }^{\mathrm{b}}$ \\ ${ }^{a}$ Klinik und Poliklinik für Hämatologie und Hämatologisches Zentrallabor, Inselspital, Bern, Switzerland \\ ${ }^{\mathrm{b}}$ Transfusionsmedizinische und Hämostaseologische Abteilung, Universitätsklinikum Erlangen, Germany
}

Therapeutic (hem)apheresis (TA) using automated blood processing equipment is a highly developed technique to selectively deplete, exchange, replace, or at least manipulate particular cellular or soluble blood constituents in a broad panel of different diseases. Starting almost 100 years ago by Abel and co-workers in 1914 [1], it still continues to expand as the pathomechanisms of new and old disease entities are elucidated, confirming 'old' indications or revealing new rationales for the application of TA. In the last decades evidencebased approaches lead to a better understanding of the clinical role of TA in the various diseases and patients, resulting in increasingly elaborated guidelines for different indication categories of TA [2]. Meanwhile TA is a well-established treatment modality for many different conditions, and it has been approved to relieve symptoms (symptomatic therapy) or even save lives. Nevertheless, the quality of evidence supporting the use of TA is often limited. Due to the rarity of many of the diseases and disorders treated by TA, prospective, randomized, controlled, clinical trials could not be performed. In keeping with this vision, TA often remains a specially tailored therapy for selected single patients who, for instance, need an emergency cell reduction to improve acute and sometimes life-threatening symptoms. The common source of information available for specific treatment approaches often relies on retrospective studies and data collections or even case reports. Nevertheless, experience of experts and their recommendations on that basis are still an important hallmark to further improve the targeted application of TA, which often is the result of an interdisciplinary approach and needs the input of different medical specialties to meet the requirements of the single patients. Therefore the interdisciplinary organization and development of the treatment planning is very challenging for the staff involved. Thus, the available experience of transfusion medicine and other medical disciplines involved in the treatment provides the basis for optimized therapy plans which could be shared with patients and clinical services requesting the use of TA.
The present issue of Transfusion Medicine AND Hemotherapy includes contributions of experts about examples of interesting indications for TA commonly evidenced by the available literature and their own experience.

First, Zeitler and colleagues [3] report about their excellent results in patients with life-threatening acquired hemophilia. Their treatment protocol includes antibody depletion through immunoadsorption, i.v. immunoglobulin treatment, immunosuppression, and high-dose factor VIII substitution. Beyond the very rapid depletion of the inhibitor (median of 3 days), they observed a long-term complete response of $100 \%$ ( $\mathrm{n}=$ 57 ) in the idiopathic autoimmune patients, when excluding the 5 paraneoplastic cases who showed a partial response. Because of this, until now, unique and surpassing outcome they recommend their protocol to be considered as a first-line treatment. The editors completely support this well-founded statement.

The second paper by Rummler and Barz [4] provides insights into the current concepts and results of TA as well as immunoadsorption in the context of thoracic organ transplantation. The authors summarize that the quick removal of antibodies and other plasma factors by plasma exchange or immunoadsorption is an effective adjunct treatment method for antibody-mediated transplant rejections and even facilitates the transplantation of patients with preformed antibodies. Finally, based on their own experience, they concluded that immunoadsorption seems to be more effective than plasma exchange in heart transplant recipients with HLA and nonHLA antibody-induced graft dysfunction.

The third contribution by Hölig and Moog [5] is a concise review of therapeutic leukocytapheresis in patients with symptomatic or threatening hyperleukocytosis. The available (only retrospective) studies have shown beneficial effects in early morbidity and mortality of patients with newly diagnosed acute myeloid leukemia without significant translation in overall long-term survival. While induction chemotherapy is the most important treatment in these patients and must

\section{KARGER \\ Fax +497614520714 \\ Information@Karger.de}

www.karger.com (c) 2012 S. Karger GmbH, Freiburg

$1660-3796 / 12 / 0394-0232 \$ 38.00 / 0$

Accessible online at:

www.karger.com/tmh
PD Dr. Behrouz Mansouri Taleghani

Klinik und Poliklinik für Hämatologie und

Hämatologisches Zentrallabor, Inselspital

3010 Bern, Switzerland

Tel. +41 31 632-8278, Fax -3406

Behrouz.MansouriTaleghani@insel.ch 
never be postponed, leukocytapheresis is recommended as an acute and effective symptomatic measure.

The following article by Leitner and Worel [6] describes two selective leukocyte adsorption systems for treatment of resistant and steroid-dependent cases of ulcerative colitis and Crohn's disease. Both systems are able to selectively remove the main mediators of the disease, particularly the activated proinflammatory cytokine-producing granulocytes, as well as monocytes and macrophages from the patient's blood circulation. Both systems provide a low-risk therapy with only few adverse reactions. Currently available studies reveal at least short-time efficacy, predominantly in patients with ulcerative colitis.

The last contribution by Worel and Leitner [7] is a sound review about extracorporeal photopheresis (ECP). ECP is a combination of leukapheresis and photodynamic therapy in which blood is treated with photoactivable drugs activated with ultraviolet light and re-infused to the patient. It is a welltolerated therapy, which has been used successfully for more than 30 years in the treatment of erythrodermic cutaneous T-cell lymphoma and for over 20 years in the treatment of chronic graft-versus-host disease (GVHD). It has also shown promising results in the multimodal treatment approaches of acute GVHD and other T-cell-mediated diseases, including systemic sclerosis, treatment and prevention of solid organ rejection, and more recently Crohn's disease.

Taken together, the editors are convinced that the manuscripts of this issue of Transfusion Medicine and Hemotherapy focusing on specific aspects of therapeutic apheresis present the experience of experts on their field and will be very valuable for the concerned units in terms of a practical and scientific support for patients' treatment planning.

\section{References}

1 Abel JJ, Rowntree LG, Turner BB: Plasma removal with return of corpuscles (plasmaphaeresis) J Pharmacol Exp Ther1914;5:625-641.

2 Szczepiorkowski ZM, Winters JL, Bandarenko N, Kim HC, Linenberger ML, Marques MB, Sarode R, Schwartz J, Weinstein R, Shaz BH; Apheresis Applications Committee of the American Society for Apheresis. Guidelines on the use of therapeutic apheresis in clinical practice - evidence-based approach from the Apheresis Applications Committee of the American Society for Apheresis. J Clin Apher 2010;25:83-177.
3 Zeitler H, Ulrich-Merzenich G, Panek D, Goldmann G, Vidovic N, Brackmann H-H, Oldenburg $\mathrm{J}$ : The benefit of extracorporeal treatment for the acute und long-term outcome of patients with lifethreatening acquired haemophilia. Transfus Med Hemother 2012;29(4):264-270.

4 Rummler S, Barz D: Plasma exchange and immunoadsorption of patients with thoracic organ transplantation. Transfus Med Hemother 2012;29(4): 234-240.
5 Hölig K, Moog R: Leukocyte depletion by therapeutic leukocytapheresis in hyperleukocytosis. Transfus Med Hemother 2012;29(4):241-245.

6 Leitner GC, Worel N: Selective granulocyte and monocyte apheresis as a non-pharmacological option for patients with inflammatory bowel disease. Transfus Med Hemother 2012;29(4):246-252.

7 Worel N; Leitner GC: Extracorporeal photopheresis. Transfus Med Hemother 2012;29(4):254-262. 